\title{
Discursos mediáticos y trabajo autogestionado: ¿Cómo se representó el proceso de recuperación de fábricas luego de la crisis del 2001 y diez años después?*
}

\author{
Media discourses and workers' self-management: How the process of \\ recovered factories was represented after 2001's crisis and ten years later?
}

Dra. Vanesa Coscia**

\section{Resumen}

En la actualidad, los medios masivos son uno de los espacios más relevantes en lo que refiere a la (re)configuración de imaginarios sociales y subjetividades. Es por esta razón que el análisis de las representaciones mediáticas sobre el mundo laboral invita a reflexionar sobre el modo en que se somete a consideración pública la problemática del trabajo en sociedades mediatizadas.

Específicamente, este artículo tiene por objetivo analizar de qué modo se otorgó visibilidad, desde los principales diarios de circulación masiva de Argentina-Clarín y La Nación-, a los procesos de ocupación/recuperación de fábricas, autogestionadas por sus trabajadores, en dos períodos, 2002/2004 y 2010/2013.

Para el abordaje metodológico de las representaciones mediáticas sobre la problemática de la recuperación de fábricas se utilizó, en este estudio, una combinación de herramientas

\footnotetext{
* Artículo recibido el 3 de Abril de 2016. Aceptado el 26 de mayo de 2016.

** Es Doctora en Ciencias Sociales y Licenciada en Ciencias de la Comunicación (Facultad de Ciencias Sociales, Universidad de Buenos Aires). Actualmente es Investigadora del Consejo Nacional de Investigaciones Científicas y Técnicas (CONICET) con sede en el Instituto de Investigaciones Gino Germani (UBA), Argentina. Correo electrónico: vanesa.coscia@gmail.com
} 
De Prácticas y discursos/ Universidad Nacional del Nordeste/ Centro de Estudios Sociales

socio-semióticas que permiten dar cuenta de las interrelaciones

discursivas y socio-comunicacionales en la superficie mediática.

\section{Palabras clave}

Representaciones mediáticas-recuperación de fábricas- trabajo autogestionado.

\section{Abstract}

At present, the mass media are one of the most important spaces in which the (re) configuration of social imaginaries and subjectivities take place. It is for this reason that the analysis of mass media's representations on the world of labor invites us to reflect on the place publicly assigned to labor issues in "mediated societies".

Specifically, the main objective of this article is to analyze the representations built by the leading Argentinean newspapers (Clarín and La Nación) on processes of occupation/recovered factories, by workers self-management, in two periods 2002/2004 and, ten years later, 2010/2013 .

The methodological approach to the media representations about recovered factories developed in this study used a combination of socio-semiotic tools that allowed us to account for the inter-discursive and socio-communication elements in the media surface.

\section{Keywords}

Mass media representations-recovered factories-workers' self-management

\section{Introducción}

Este artículo tiene por objetivo mostrar de qué modo se otorga visibilidad, desde los principales medios gráficos de circulación masiva de Argentina-Clarín y La Nación-,a los procesos de ocupación/recuperación de fábricas, autogestionadas por sus trabajadores. Para ello, se identificaron dos períodos claves en estas experiencias: el primero en el 2002/2004, los años inmediatamente posteriores al estallido de la crisis 
De Prácticas y discursos/ Universidad Nacional del Nordeste/ Centro de Estudios Sociales

del 2001, cuando hace eclosión la ocupación de fábricas en el país; el segundo,diez años más tarde, cuando las fábricas recuperadas con sus avances y retrocesos continúan en marcha y, a su vez,nacen nuevas experiencias de recuperación.

Las preguntas que surgen en esta investigación,y que se intentarán responder a lo largo de este artículo, son: ¿Cuáles fueron las "lecturas preferenciales" que se pusieron en juego a la hora de representar estos procesos? ¿Les fue asignada una atribución positiva o negativa a estas experiencias? ¿De qué modo se las legitimó o deslegitimó? ¿Cómo fue variando su representación-si lo hizo- en los diversos contextos mencionados? ¿Cuáles son los sentidos que se vehiculizan desde las páginas de los medios sobre la noción de "trabajo autogestionado" en el marco de estas experiencias?

Este trabajo tiene sus antecedentes en investigaciones previas centradas en el análisis interdisciplinario de los Estudios Culturales, en particular, sobre los medios masivos de comunicación y la problemática de los estudios del trabajo. En dicha intersección se han venido analizando, anteriormente,representaciones mediáticas del conflicto laboral y de reformas del mercado de trabajo en Argentina en diversos momentos históricos -fines de los '80, luego del $2004^{-1} \mathrm{y}$ también se realizaron comparaciones con otros países comoItalia(Coscia,2013), con el que Argentina comparte un sistema de medios fuertemente concentrado y centralizado en pocas manos $^{2}$. Específicamente, en el presente artículo, más que preguntarse por el modo en que seconstruye la noticia sobre una huelga clásica o las atribuciones asignadas a los representantes sindicales de las principales confederaciones, nos interrogamos sobre el modo en que el proceso de ocupación y posterior recuperación de fábricas fue representado en las páginas de Clarín y La Nación o, en otras palabras, en qué modo adquirió relevancia pública dicha temática.

Conceptualmente, se consideran los aportes de Gramsci (1974) para reflexionar sobre el rol de los medios masivos en tanto co-constructores de hegemonía cultural,

\footnotetext{
${ }^{1}$ Para ver detalladamente consultar Coscia, 2011 y 2008.

${ }^{2}$ Para ampliar sobre la cuestión de la concentración de medios -y sus implicancias sociales y políticas- en Argentina, ver Mastrini y Becerra, 2006; para profundizar en la concentración a nivel europeo, ver Doyle,2008.
} 
De Prácticas y discursos/ Universidad Nacional del Nordeste/ Centro de Estudios Sociales

social y política en las actuales sociedades mediatizadas (Verón, 1987). En esa misma línea de pensamiento, se retoma la perspectiva de Hall (1984) acerca de concebir a la comunicación y a la cultura como un terreno de disputa simbólica donde no hay victorias definitivas y siempre hay posiciones estratégicas que se conquistan y se pierden. A partir de ello, se sostiene que los grandes medios masivos son actores políticos (Borrat, 1989) que, bajo el paraguas de la llamada "neutralidad", gozan de la ventaja de ser pensados como imparciales, mientras en realidad se trata de grupos de poder que intentan incidir en la sociedad en cada momento histórico.

La hipótesis inicial que guía esta investigación, por lo tanto, es que el tratamiento mediático de situaciones de crisis y conflictos específicos relacionados con el mundo del trabajo es permeable al contexto político, económico y cultural en el que dicha conflictividad se expresa.

Para abordar el análisis empírico de los discursos mediáticos se realizó una búsqueda exhaustiva de las noticias sobre la temática en los dos diarios nacionales de mayor tirada de Argentina (Clarín y La Nación), en los dos períodos mencionados (2002/2004 y 2010/2013). Luego, se utilizó una combinación de herramientas sociosemióticas que permite dar cuenta de las interrelaciones discursivas y sociocomunicacionales en la superficie mediática. El enfoque socio-semiótico plantea el análisis de los fenómenos sociales tanto desde la dimensión significante como desde las condiciones sociales de producción de los discursos, entendiendo la producción de sentido como una práctica social que permite comprender la producción de subjetividades (Verón, 1987). Se trata, parafraseando a Van Dijk (1997), de identificar la delimitación de un "campo de significados favoritos" en torno a la construcción de las noticias sobre el tema de la recuperación de fábricas. Al conjunto de noticias seleccionadas se le aplicó una matriz analítica de medios gráficos puesta a prueba en trabajos previos.Dicha matriz contiene tres dimensiones: Formal, permite dar cuenta de lo paratextual y contextual de la noticia: la ubicación en secciones, los títulos, la inclusión de fotos, los géneros noticiosos -crónica, nota de opinión, editorial;Contenidos, importancia de la construcción del texto noticioso, temáticas privilegiadas, 
De Prácticas y discursos/ Universidad Nacional del Nordeste/ Centro de Estudios Sociales

criterios de noticiabilidad, virajes en la representación; Campo de interlocución, fuentes, "actores" de la noticia, contrato de lectura. ${ }^{3}$

El corpus del primer período coincide con el momento inmediatamente posterior a la crisis del 2001, en el que se produce el mayor crecimiento de este tipo de experiencias de recuperación de fábricas. En efecto, como afirma Ruggeri (2014), en el período que va desde el 2001 y hasta el 2004 se conformaron 161 empresas recuperadas por sus trabajadores en el país y la mayor parte de ellas nació como respuesta a la crisis del 2001. Según el autor, "a medida que se estabiliza la situación -de crisis-, la curva va descendiendo hasta estabilizarse en pocos casos por año" (2014:15).

Luego del 2004 y hasta la actualidad se crearon lo que Ruggeri denominó "nuevas recuperadas", entre las que se contabilizan poco más de 140 hacia diciembre de 2013 y que no necesariamente surgieron como respuesta a una crisis. En este punto, se coincide conPalomino et al. (2011) en que mientras en las primeras experiencias el origen de la recuperación constituyó una respuesta no convencional de los trabajadores ante la crisis, en el caso de las nuevas experiencias, la recuperación opera ya sobre un mecanismo cuasi-institucional que permite evitar el cierre. En ese sentido, afirma el autor, la recuperación de empresas se ha transformado en una estrategia de lucha viable y conocida por los trabajadores. Además, el período 2010/2013 -segundo período seleccionado para el análisis-coincide con un incremento cuantitativo de casos de recuperación de fábricas ${ }^{4}$.

Es importante señalar que, tal como afirma Perbellini (2014), estas empresas conforman "un espacio social común creado por los trabajadores, constituido en torno a un conocimiento productivo anterior que refleja una historia común como colectivo de trabajo". En ese sentido, como señala la autora, los trabajadores ejercen una

\footnotetext{
${ }^{3}$ Para ver en forma detallada cada uno de estos elementos y su relevancia a la hora de realizar análisis de discursos en prensa gráfica masiva, ver Coscia, 2011.

${ }^{4}$ Efectivamente, del 2010 al 2013 se crean 63 nuevas empresas recuperadas, de las cuales se registran 41 casos solo en 2012/2013. Para ampliar la cantidad, distribución y evolución de los casos, consultar el "Informe del IV relevamiento de Empresas Recuperadas en la Argentina" del Programa Facultad Abierta, Facultad de Filosofía y Letras del 2014.
} 
De Prácticas y discursos/ Universidad Nacional del Nordeste/ Centro de Estudios Sociales

"actividad creativa" que permite la puesta en funcionamiento de una unidad productiva gestionada por los mismos trabajadores dentro de un sistema capitalista.

Al partir de esta conceptualización del fenómeno, si bien este trabajo focaliza en el análisis de las representaciones mediáticas sobre el proceso de empresas recuperadas -y no sobre las características que adquiere este proceso-, resulta fundamental conceptualizarlo para no perder de vista el análisis de "las ausencias", es decir, aquello que se omite, se oculta o no se visibiliza en las noticias que tematizan este tipo de experiencias. Como asevera Bourdieu, "pensar de modo estructural nos obliga a percibir la importancia de las ausencias" (1990: 271).

\section{Cuando se somete a consideración pública la recuperación de fábricas}

En primer lugar, en lo que respecta al nivel de ventas de los diarios, se produjeron algunos cambios entre los períodos de análisis seleccionados (2002/2004 y 2010/2013). Según el Instituto Verificador de Circulaciones (IVC), la tirada de Clarín hacia el 2001 era de poco más de 450.000 ejemplares vendidos en promedio de lunes a domingo en todo el país, hacia el 2008 descendió a poco menos de 380.000 y continúo con su curva descendente para llegar, en el 2013, a una tirada de 250.000 ejemplares. No obstante esta disminución, continuó siendo el matutino más vendido -según el IVC-, seguido de La Nación, el segundo matutino en ventas ${ }^{5}$. El diario La Nación hacia el 2013 contaba con una tirada promedio de poco más de 160.000 ejemplares en todo el país, pero su nivel de ventas no se modificó marcadamente entre 2001 y 2013, a diferencia de Clarín 6 .

\footnotetext{
${ }^{5}$ Los diarios Página12 y Tiempo Argentino, con una ideología acorde a la del gobierno kirchnerista, no están inscriptos en el IVC. Por lo tanto, se desconoce a nivel de datos oficiales su tirada anual promedio. Sin embargo, fuentes no oficiales, como la empresa publicitaria PautaMás que, entre otros servicios, vende espacios de publicidad en diarios y revistas nacionales, señala en la actualidad una tirada aproximada de Página 12 de 20.000 ejemplares y 70.000 ejemplares para Tiempo Argentino.

${ }^{6}$ En efecto, entre el 2001y 2013, el diario La Nación osciló entre 150.000 y 170.000 ejemplares vendidos a nivel nacional de lunes a domingo, según el IVC. En el 2001 contaba con una tirada promedio de 170.000 ejemplares; en el 2008,de 158.000 y en el 2013,de160.000.
} 
De Prácticas y discursos/ Universidad Nacional del Nordeste/ Centro de Estudios Sociales

Para explicar, en parte, esta disminución, es preciso señalar que a partir del 2008 se evidenció una fuerte confrontación entre los grandes grupos multimediales argentinos y el gobierno de Cristina Fernández de Kirchner, que se expresó en cuestiones como el "conflicto del campo", "Fútbol para todos", "Papel Prensa" y la sanción de Ley de Servicios de Comunicación Audiovisual 26522/9-que reemplazaba a la Ley $22.285 / 81^{7}$.Todo lo cual permite entender, además, en qué modo pudo haber incididodicho contexto "beligerante" en algunas de las representaciones que se construyeron sobre las empresas recuperadas en el período 2010/2013.

En un primer acercamiento al corpus de noticas seleccionado, es posible observar que la visibilidad otorgada a la temática-noticias que tematizan de manera central las recuperaciones de fábricas- por los principales diarios de tirada nacional Clarín y La Nación- es mayor en el período 2002/2004 que durante2010/2013 (se relevaron 67 noticias para el primer período -41 de Clarín y 26 de La Nación- y 45 noticias en total para el segundo período -17 de Clarín y 28 de La Nación-). Respecto de la cantidad total de noticias relevadas (112),es importante resaltar la notoria disminución en la cobertura de la temática del diario Clarín durante el segundo período. En efecto, mientras que La Nación mantuvo un promedio de 26/28 noticias en cada momento,Clarín durante 2002/2004 puso en circulación más de 40 noticias y tan solo 17 en el segundo período de análisis.

Al partir de estos datos cuantitativos, pero sin reducir la problemática de la visibilidad solo a la cantidad de noticias seleccionadas, en este estudio nos centramos, como se dijo previamente, en identificar también aquello que se resalta o se omite en las coberturas mediáticas de ambos períodos, teniendo en cuenta las características que adquiere la representación en cada momento y los virajes -si existieron- en el modo de someter a consideración pública la problemática de la recuperación de

\footnotetext{
${ }^{7}$ Como señala Becerra, la difícil aplicación de dicha ley profundizó el enfrentamiento entre el gobierno y los multimedios; y aun así no se logró superar el proceso de concentración. No obstante, fue precedida por un hecho inédito en la historia del sistema de comunicaciones en Argentina: "Un debate masivo sobre la función de los medios, sus intereses y alineamientos" (2015: 38). El pasado 4 de enero de 2016, el gobierno de Mauricio Macri estableció por Decreto de Necesidad y Urgencia (DNU) modificaciones a dicha ley que implican un fuerte retroceso respecto del intento previo de desconcentración mediática. Ver dicho texto en el Boletín Oficial.
} 
De Prácticas y discursos/ Universidad Nacional del Nordeste/ Centro de Estudios Sociales

fábricas y del trabajo autogestionado en Argentina. En ese sentido, se tiene en cuenta la diferenciación planteada por Martini (2000) entre agenda temática-el tema de la noticia-, que se refiere a los problemas o temas que un medio considera relevantes incluir, y agenda atributiva-el modo de construir las noticias-, que se refiere a los atributos que el medio selecciona para caracterizar a temas, personajes o tipos de acontecimientos.

\subsection{Crisis del 2001, fábricas que cierran y trabajadores que ocupan}

Como señalaronalgunasinvestigaciones previas (Palma, 2004; Settani, 2005), la representación mediática de los movimientos de desocupados o "piqueteros" se realizó a partir de la deslegitimación del accionar piqueteroa través de diversos mecanismos discursivos que le asignaban la atribución de "violentos" e "intimidantes", con una metodología de lucha peligrosa;y luego del 2003, en un período de "normalización institucional"(Svampa, 2005), se enfatizó desde los grandes medios que dicha metodología estaba desgastada ${ }^{8}$.

Esas caras que no se dejan reconocer, donde sí puede reconocerse la vigencia intimidatoria de consignas y conductas que atribuyen una función redentora (...) sobre todo a la violencia.

(Nota titulada "El riesgo de ignorar lo que sucede", sección Política, La Nación, domingo 27 de junio de 2004).

Titular: Volvieron los cortes pero la protesta fue más débil.

Epígrafe de foto: Raleado. El corte de ayer en la ruta 3 en La Matanza. Se improvisó una asamblea para discutir el futuro de este método de protesta.

\footnotetext{
${ }^{8}$ En efecto, Svampa (2005) señala en sus investigaciones que luego de 2003se intentó generar un consenso público "antipiquetero", en donde los sindicatos comenzaban a ser la referencia "legítima" para motorizar los conflictos relacionados con la problemática del mercado de trabajo.
} 
De Prácticas y discursos/ Universidad Nacional del Nordeste/ Centro de Estudios Sociales

(Clarín, El País, 14 de agosto de 2004 -el resaltado es del diario).

Esto se realizó desde diversos mecanismos discursivos que dieron un sentido general a la noticia: en el primer caso poniendo el acento en la violencia y la intimidación como dos rasgos "esenciales" de los piqueteros. Además, colocaron la noticia en una de las secciones más importantes del diario -sección política- y en uno de los días con más tiempo y tranquilidad para leerlo-domingo-. En la segunda cita se puede apreciar de qué modo los elementos de titulación indican la forma de leer la imagen fotográfica, el epígrafe que refuerza dicha dirección -el corte fue raleado- y la inclusión de pequeñas notas de opinión como aquella titulada "desgastes" que funcionan como resumen de la nota principal y destacan aquello que el diario considera central en la información -la debilidad del movimiento piquetero-. A partir de estos modos de construir las noticias, se consolidaba, en la superficie mediática, un estereotipo negativo del "piquetero" en este período.

Paralelamente, dichas atribuciones negativas se asociaron, en la representación, a las de "asistencialismo" a través del pedido de subsidios y planes al gobierno para los desocupados.

Piqueteros lanzaron una nueva ofensiva contra el Gobierno (...) Los funcionarios rechazaron una vez más la entrega de 3000 subsidios (...) Molestos con la nueva negativa para conseguir los subsidios, los piqueteros que anoche salieron de Trabajo denunciaron que el Gobierno "está por desatar una persecución injusta...".

(La Nación, Opinión, 29 de octubre de 2003).

En este sentido, es posible coincidir con Baquero (2006:9) en que estos tipos de notas de opinión "cumplen una función metadiscursiva, comentan los sucesos construidos como noticias y operan como escenarios de disputa política por imponer modelos de un país". 
De Prácticas y discursos/ Universidad Nacional del Nordeste/ Centro de Estudios Sociales

En ese contexto, frente al permanente pedido de ayuda al gobierno de los piqueteros "violentos", la contracara fue mostrar "positivamente" las nuevas formas de asociación de trabajadores, entre ellas, los clubes de trueque o las empresas recuperadas que ponían nuevamente a funcionar sus trabajadores con esfuerzo y sacrificio.

Experiencia en pleno crecimiento (...) El primer club del trueque se fundó en Bernal, a principios de 1995 (...) A fin de año ya eran 60.

(Clarín, Sociedad, domingo 3 de marzo de 2002).

Herramientas de participación y control popular (...) eso es lo que se está pariendo en los argentinos involucrados en asambleas, fábricas recuperadas, en experiencias de autogestión comunitarias.

(Clarín, Suplemento Zona, domingo 26 de enero de 2003).

Este modo de representar las nuevas asociaciones de trabajadores o las alternativas de "volver al trabajo" -en la mayoría de los casos también con amplias coberturas, publicadas el domingo y, por lo tanto, otorgando una alta visibilidad a la temática- pusieron el acento en los procesos de "autogestión" como elemento clave para reinsertarse en el mercado de trabajo en tiempos de crisis:

Autogestión: un auxilio al empleo (...) Hay unas 10.000 personas que viven de su tarea en empresas administradas por sus trabajadores.

(Clarín, Economía, 23 de diciembre de 2002).

Autogestión: el camino para retomar la producción (...) Puertas que se van abriendo

(La Nación, Comercio Exterior, 29 de octubre de 2003). 
De Prácticas y discursos/ Universidad Nacional del Nordeste/ Centro de Estudios Sociales

La otra cara de la crisis económica (...) Tras la quiebra, las empresas cerraban sus puertas, las máquinas se paraban y una lista de obreros se sumaba a la cola de desocupados. Pero durante los últimos años los trabajadores comenzaron a revertir ese proceso. Tomaron en sus manos las fábricas, las abrieron nuevamente y las pusieron a funcionar en forma de cooperativas controladas por los obreros

(La Nación, "Fabricas que producen cultura", Espectáculos, 6 de diciembre de 2003).

A partir de ello, sería posible afirmar que en la superficie mediática durante el período 2002/2004 se construye la oposición asistencialismo piquetero/trabajadores autogestionados, asignándoles a los primeros las atribuciones negativas de peligrosidad, debilidad y asistencialismo estatal ${ }^{9}$. En cambio, a los segundos se les asignaron las atribuciones positivas de "abrir puertas" y "tomar en sus manos las fábricas", conceptos que lejos de disturbar la paz social -como lo propugnaba la 'amenaza' piquetera-, contribuían a recomponerla, salvando las fuentes de trabajo, a través de la autogestión.

Los trabajadores que consiguieron salvar empresas quebradas (...) Ya hay casi 60 empresas en el país que funcionan a fuerza del trabajo de sus empleados. Así lograron conservar cerca de 4000 puestos laborales.

(Clarín, Sociedad, domingo 17 de marzo de 2002).

Cintillo de nota: Una salida a la crisis|En Capital y Gran Buenos Aires. Titular: Crecen los casos de empresas recuperadas por empleados.

(Clarín, La Ciudad, 10 de octubre de 2003).

\footnotetext{
${ }^{9}$ Es importante destacar que si bien entre los reclamos de los piqueteros también estaban los pedidos al gobierno de "trabajo genuino", y no sólo de planes, en las noticias relevadas en este período se resalta, principalmente, la noción de "pedido de asistencia al gobierno".
} 
De Prácticas y discursos/ Universidad Nacional del Nordeste/ Centro de Estudios Sociales

Otra de las nociones positivas que recorren las representaciones de las empresas recuperadas en Argentina post 2001, unidas a la de idea de autogestión, a través del sacrificio y del esfuerzo, es la noción de solidaridad, sea al interior de los nuevos espacios de trabajo, sea en relación con otros ejemplos de nuevas empresas autogestionadas.

Ahora no hay patrones. Los 45 operarios se reparten en todas las funciones y recorren las maquinarias y pasillos con distensión poco habitual en una fábrica. (Clarín, Sociedad, 29 de agosto de 2002).

Una red de ayuda (...) Las cooperativas de trabajo que recuperaron empresas formaron una red informal de ayuda entre ellas.

(Clarín, Sociedad, domingo 17 de marzo de 2002).

El espíritu solidario que reina en ellas [las cooperativas] restablece en parte no sólo una cultura del trabajo autogestionada sino también la autoestima de miles de argentinos.

(Clarín, Economía, domingo 6 de octubre de 2002).

Aquí puede verse que los sentidos que recorren la "autogestión" de estas empresas se asocian a las nociones de ayuda, solidaridad y cooperación en el contexto de una nueva modalidad de trabajo "sin patrones". A su vez, con ello se pone en relación la idea de elevación de autoestima o, en otras palabras, de la dignidad que da el trabajo. A esta dignidad se suman también los conceptos de "creatividad" y "reinvención" para hacer frente a un mercado laboral en crisis:

Fábricas de las cenizas (...) La Argentina que reinventa el trabajo (...) La profundización de la crisis económica fogoneó el más nuevo de los fenómenos sociales: la recuperación en manos de los trabajadores de las fábricas cerradas. (Clarín, domingo 22 de septiembre de 2002, el subrayado es propio). 
Con creatividad se puede superar la crisis (...) La fábrica de cerámicas Zanon volvió nuevamente a la actividad gracias a la autogestión realizada por sus trabajadores

(La Nación, Suplemento Arquitectura, 15 de enero de 2003, el subrayado es propio).

A partir de ello, en la superficie mediática, el trabajo sin patrones, la autogestión de los trabajadores de las fábricas recuperadas fuemostrada "positivamente" $y$, en ese sentido, es posible afirmar fuelegitimada por los grandes medios nacionales que frente al mundo de los piqueteros "peligrosos" y que buscaban vivir de subsidios estatales, la ocupación de una fábrica era una alternativa viable, "creativa" y "digna" de volver al trabajo, luego del estallido de la crisis del 2001.

\subsection{Las recuperaciones, diez años después}

Tal como se mencionó anteriormente, luego del 2004, con la llegada de las "nuevas recuperadas", se asiste a un cambio en el modo de concebir dichos procesos sociales y laborales, dado que aquí las empresas recuperadas por sus trabajadores no se asocian tanto a "respuestas desesperadas frente a crisis económicas generalizadas", sino a "una variedad de circunstancias críticas que no necesariamente deben contextualizarse en situaciones de grandes crisis de carácter estructural" (Ruggeri, 2014: 7). Al partir de dicha conceptualización, en este apartado se pretende identificar sieste cambio en la concepción de las empresas recuperadas impactó de alguna manera en los modos de dar visibilidad a la temática, desde los grandes medios, y cuáles fueron los virajes en las representaciones -si es que se produjeron- en el marco de un contexto económico, político y social también diverso.

Para el período 2010/2013 se relevaron 45 noticias publicadas en los diarios Claríny La Nación, de las cuales solo 17 corresponden a Clarín y 28 a La Nación. En este caso, la cantidad de noticias publicadas porLa Nación no sólo es mayor, sino que 
De Prácticas y discursos/ Universidad Nacional del Nordeste/ Centro de Estudios Sociales

también es más extendida y diversificada en sucobertura. Es decir, este diario le otorga más espacio, más jerarquización-notas publicadas los sábados y domingos-, con una mayor diversidad en los géneros de las noticias incluidas -editoriales, análisis, columnas de economía, carta de lectores-que Clarín. Por su parte, en Clarín se reduce notablemente la cantidad de noticias que tematiza estas cuestiones y los espacios otorgados -en general pequeños recuadros- están pocos jerarquizados.

En el contexto post 2010, es posible observar que la temática es presentada desde estos medios de manera "positiva", en tanto se trate de casos "productivos" y "exitosos".

Una fábrica recuperada es la mayor productora de tractores.

(Clarín, Suplemento Agronegocios, 26 de noviembre de 2013).

La fábrica recuperada Industria Metalúrgica y Plástica Argentina (IMPA), en Almagro, es un crisol de actividades productivas y culturales. Desde 1998, tras la quiebra, los propios trabajadores tomaron en sus manos la producción y en la actualidad se ocupan de fabricar tubos de dentífrico en aluminio y bandejas para comida de avión.

(Clarín, Suplemento Arquitectura, 10 de enero de 2013).

Se ha vuelto común enterarse de casos exitosos llevados adelante por cooperativas, empresas recuperadas.

(La Nación, Suplemento Comunidad, sábado 2 de junio de 2012).

Sin embargo, y teniendo en cuenta el contexto beligerante post 2008 entre el gobierno y los multimedios, señalado anteriormente, en forma paralela al mencionar algunos casos exitosos, aquello que se resalta en la superficie mediática es la "ayuda estatal" para el efectivo funcionamiento de estas empresas. En ese sentido, se incluyen noticias que hacen hincapié en que el gobierno kirchnerista subsidia a estas empresas recuperadas y a cooperativas de trabajo. 
De Prácticas y discursos/ Universidad Nacional del Nordeste/ Centro de Estudios Sociales

La cooperativa Encata, una fábrica recuperada de Catamarca que fue reinaugurada en febrero de 2011 por la presidenta Cristina Kirchner, comenzará a producir sus envases flexibles (...) Encata cuenta con 23 empleados, que reciben un subsidio nacional.

(La Nación, Columna económica "qué pasa", 20 de agosto de 2012).

Las empresas señaladas lo hacían arropadas por el aliento oficial, planes como Argentina Trabaja y programas como el Fondo del Bicentenario, entre otros. El empresario casi asumía un papel de reparto, de mero coordinador de todos esos recursos oficiales para llevar a buen puerto la potencia creadora de empleos.

(La Nación, Opinión, 2 de agosto de 2012, el subrayado es propio).

A partir de esta última nota de opinión, y retomando nuevamente a Baquero (2006) sobre los intentos de los medios por imponer "modelos de país", en este caso es posible identificar que "el modelo" para La Nación supone que los recursos oficiales no deberían ser malgastados. Esta crítica a la utilización de recursos públicos puede verse, además, en la inclusión de la voz de los mismos lectoresque se expresan al respecto en las páginas del diario:

La mayoría de las empresas recuperadas no pagan impuestos; son sostenidas con cuantiosos subsidios por los oficialismos.

(La Nación, Sección Carta de lectores, 6 de junio de 2011, el subrayado es propio).

Como puede observarse en la cita precedente, a través de esta carta de lectores, el mismo diario expresa su postura. En efecto, tal como afirma Tuchman (1986), la inclusión de ciertas voces o fuentes tiene la ventaja de poner en boca de otro las opiniones del periodista, consiguiendo que otros digan lo que ellos mismos piensan.A 
De Prácticas y discursos/ Universidad Nacional del Nordeste/ Centro de Estudios Sociales

través de este mecanismo discursivo, el medio criticalos usos que se hacen de los recursos del Estado, en este caso subsidiando a empresas recuperadas.

Entonces, si en el período 2002/2004 fueron los piqueteros representados como aquellos que buscaban "asistencialismo" y que querían "vivir del Estado", oponiéndose en la superficie mediática asistencialismo piquetero/trabajadores autogestionados; en el 2010/2013, para los medios, son las mismas empresas recuperadas las que se vuelven "asistidas" por el Estado. Esta representación tiene, a su vez, una contrapartida: estas mismas cooperativasfuncionan como un lugar para "recabar votos" y se colocan militantes oficialistas a trabajar en ellas.

Se trata de una clara maniobra electoral (...) Por un lado, los intendentes del conurbano bonaerense continúan recibiendo beneficiarios del Plan Argentina Trabaja a través de cooperativas que ellos mismos conforman (...) Por el otro, el Movimiento Evita, desde el gobierno bonaerense y con recursos que le derivará la Nación, continúa conformando cooperativas y controlará a la totalidad de las cooperativas de trabajo.

(La Nación, Editorial, 3 de septiembre de 2013).

En el mismo sentido,Clarín, en este caso desde su sección política, estigmatiza dicha "asistencia" no sólo por el desgaste de los recursos públicos, sino además por maniobras "turbias" de los funcionarios kirchneristas.

La Legislatura bonaerense expropió de manera irregular la fábrica SIAM, que estaba en manos de una cooperativa de trabajadores y ahora será controlada por un empresario ligado al kirchnerismo.

(Clarín, Política, 30 de enero de 2013).

El acento en este vínculo con el gobierno kirchnerista se jerarquiza en la superficie mediática a través de diversos mecanismos discursivos. En primer lugar, a través de la inclusión de editoriales. Según Borrat (1989), comentar un tema -a través de una nota de opinión o de una editorial- es conferirle un rango más elevado que el 
De Prácticas y discursos/ Universidad Nacional del Nordeste/ Centro de Estudios Sociales

de aquellos temas que solamente son narrados. En segundo lugar, a través de una multiplicidad de noticias de diferente género que otorga una mayor visibilidad a la cuestión -opinión, editoriales, crónicas, carta de lectores, etc.- en las que, a través de diferentes voces,se hace hincapié en el vínculo entre empresas recuperadas y el gobierno kirchnerista. En este punto, es preciso aclarar que la utilización del recurso de incluir voces variadas es lo que Luchessi y Cetkovich (2007) denominaron polifonía regulada. Dicho concepto, en tanto dispositivo de enunciación, se refiere a la estrategia a través de la cual los medios masivos neutralizan la heterogeneidad constitutiva del discurso, apelando a fuentes diversas para reforzar un único discurso que, en este caso, intenta deslegitimar a las empresas recuperadas por su vinculación con el kirchnerismo. En tercer lugar, la mayor parte de este tipo de noticias en la que se resalta el subsidio estatal vinculado con las cooperativas es ubicada en las secciones "duras" (Martini,2000) y, por lo tanto, de mayor importancia de los diarioscomo son política y economía.

Si bien al interior de las empresas recuperadas el vínculo con el Estado es una fuerte discusión entre los trabajadores y la necesidad de financiamiento provoca una serie de controversias en torno a las posibilidades de pedir créditos -cuando es posible-o de recibir subsidios estatales, en la superficie mediática el mecanismo discursivo que se utiliza es el de tomar, metonímicamente, la parte -la empresa subsidiada- por el todo -las empresas recuperadas en general-. En este modo de construcción se daría a entender que la totalidad de las empresas recuperadas o cooperativas reciben ayuda del gobierno kirchnerista.

En esta misma línea de análisis, cuando los medios se refieren a la situación jurídica de las empresas recuperadas, ponen nuevamente el acento en el subsidio estatal y no en la tranquilidad que puede dar a los trabajadores un marco legal seguro.

La iniciativa modifica la ley de quiebras para facilitar y legalizar el sistema de fábricas recuperadas (...) En ese sentido, habilita al Estado a darles a las cooperativas apoyo técnico y económico -en otras palabras, a subsidiarlas-, para que puedan poner en marcha sus emprendimientos. 
De Prácticas y discursos/ Universidad Nacional del Nordeste/ Centro de Estudios Sociales

(La Nación, Economía, 1 junio de 2011).

A partir de ello, es posible afirmar que el proceso de recuperación de fábricas que había sido representado muy positivamente para estos diarios en el 2002/2004, en este período es un proceso que se sostiene gracias a la ayuda del kirchnerismo y, por lo tanto, en ese punto pierde su atribución "positiva" en tanto en las páginas de los medios aquello que se resalta es que : a) se gastan-o malgastan- recursos del Estado, y b) las cooperativas o empresas recuperadasfuncionan porque terminan siendo subsidiadas por el kirchnerismo. Paralelamente, en este período la figura del "trabajador" aparece opacada, perdiendo su centralidad en el proceso.

Por otra parte, y teniendo en cuenta también aquello que no se explicita, en las notas analizadas tampoco se hace referencia a los conflictos o contradicciones que enfrentan los trabajadores que forman una cooperativa o aquellos que hace más de diez años vienen autogestionando la producción en su fábrica. Este tipo de cuestiones no son temáticas privilegiadas en las coberturas de los principales matutinos.

\section{Reflexiones finales}

Tal como se ha visto a lo largo del artículo, y retomando la hipótesis inicial, es decir, que el tratamiento mediático de situaciones de crisis y conflictos específicos relacionados con el mundo del trabajo es permeable al contexto político, económico y cultural en el que dicha conflictividad se expresa, es posible afirmar que los principales diarios de Argentina asignaron atribuciones "positivas"y "negativas" al fenómeno de las empresas recuperadas, legitimando o deslegitimando dicho procesosegún el período analizado y también según sus propios posicionamientos político-ideológicos en la cuestión.

De esta manera, es posible afirmar que en el período 2002/2004 los grandes medios apoyaron la modalidad de recuperación de fábricas a través de la alta visibilidad otorgada en sus coberturas. A partir de las 67 noticias analizadas que tematizaron la problemática, fue posible advertir que dicha tematización también se 
De Prácticas y discursos/ Universidad Nacional del Nordeste/ Centro de Estudios Sociales

correspondió con un alto grado de jerarquización. Esto se realizó a través de diversos mecanismos discursivos: por un lado, colocando las noticias en las secciones principales de los diarios, muchas de ellas el sábado o domingo -el día con más tiempo para la lectura de diarios- y, por el otro, con diversas tipologías de noticias -crónicas, notas de opinión, editoriales-. Efectivamente en Argentina, en el período 2002/2004, al proceso de recuperación de fábricas se le asignó una atribución "positiva" en tanto esfuerzo y coraje de los trabajadores por salvar su fuente de trabajo, en un momento de crisis post 2001- en el que se buscaba recomponer el tejido social y donde "volver a trabajar" era la salida "viable" y "digna". En ese sentido, fue legitimada la misma noción de "autogestión"de los trabajadores, la cualfue asociada a los valores de solidaridad, creatividad y dignidad en la superficie mediática. Por oposición, se estigmatizó y deslegitimó a los movimientos de desocupados o piqueteros, asignándoles una atribución "negativa" al calificarlos de "violentos", con una metodología "desgastada" que alteraba la paz social y enfatizando que buscaban vivir de subsidios estatales.

En el período 2010/2013, en primer lugar es importante señalar que la cantidad de noticias publicadas disminuyó respecto al período anterior, siendo dicha disminución más notoria en Clarín que en La Nación. En segundo lugar, la atribución "positiva" otorgada por los diarios al proceso de recuperación de fábricas se redujo a mencionar algunos casos "de éxito" de este tipo de experiencias. Sin embargo, dichas menciones fueronacompañadas en la superficie mediática por una atribución "negativa" que vinculaba a estas empresas recuperadas o cooperativas de trabajo con una suerte de asistencialismo cooperativo estatal.Entonces, si en 2002/2004 la noción de asistencialismo se vinculaba a los piqueteros, en este período dicha atribución "negativa"pasa a vincularse con las empresas recuperadas, y se pone el foco en que el proceso cooperativo se sostiene con recursos públicos.En el contexto del enfrentamiento entre los multimedios y el gobierno post 2008, la temática de la recuperación de fábricas parece reducirse a una disputa política: cuánto reciben de ayuda estatal y en qué medida responden a los intereses del gobierno de Kirchner.

A su vez, la representación del período 2002/2004 sobre la recuperación de fábricas, que colocaba de forma "positiva" en el centro de la escena a los trabajadores 
De Prácticas y discursos/ Universidad Nacional del Nordeste/ Centro de Estudios Sociales

autogestionados, quienes con coraje y esfuerzo llevaban adelante la producción en las fábricas-presentados casi como "héroes" que salvaban su fuente de trabajo-, en el período 2010/2013 la figura del "trabajador" aparece opacada, perdiendo su carácter de actor central en la superficie mediática. En efecto, en este último período ya no se destacan las nociones de solidaridad, creatividad y/o dignidad, tan jerarquizadas en el primer momento. Y tampoco resulta "noticiable" o atractivo para estos medios dar cuenta de los desafíos y contradicciones que enfrentan los trabajadores en este tipo de experiencias, con el correr del tiempo, en el contexto de un capitalismo cada vez más salvaje.

\section{Bibliografía}

- Baquero, R. (2015).Discursos sobre 'linchamientos': entre el repudio y la legitimación de la violencia homicida. Ponencia presentada en las XI Jornadas de Sociología. Universidad de Buenos Aires, Buenos Aires, 13 al 17 de julio (paper).

- Becerra, M. (2015).De la concentración a la convergencia. Política de medios en Argentina y América Latina. Buenos Aires: Paidós.

- Borrat, H. (1989).El periódico, actor político. Barcelona: Gustavo Gili.

- Bourdieu, P. (1990).La huelga y la acción política,Sociología y Cultura.México: Grijalbo, pp 265-276.

- Coscia, V.(2008).La privatización como salida a la crisis de fines de los '80. Un análisis desde las estrategias mediáticas,Papeles de trabajo, 4. Buenos Aires: Instituto de Altos Estudios Sociales (IDAES).

- Coscia, V. (2011).Imágenes sindicales en el principal diario Argentino: un análisis de las dinámicas mediáticas ante el 'resurgimiento' del actor gremial 2004-2007. Tesisdoctoral, Facultad de Ciencias Sociales, Universidad de Buenos Aires (inédita).

- Coscia, V. (2013). Reformas y protestas laborales en Italia: ¿Qué visibilidad y para qué?, en Wolikow, S.(ed.)Grèves et conflits sociaux. Approches croisées de la 
De Prácticas y discursos/ Universidad Nacional del Nordeste/ Centro de Estudios Sociales

conflictualité.,Dijon: Maison des Sciences del Homme, Universidad de Borgoña,pp.357-364.

- Doyle, G.(2008). Introduzione all'economia dei media. Milano: Ulrico Hoepli Editore.

- Gramsci, A.(1974).Literatura y Cultura Popular, Tomo I. Buenos Aires: Cuadernos de Cultura Revolucionaria.

- Hall, S. (1984).Notas sobre la deconstrucción de lo popular, en Samuel, R.(ed.)Historia popular y teoría socialista. Barcelona: Crítica,pp.93-110.

- Luchessi, L.y Cetkovich Bakmas, G.(2007).Mercado cultural e industrias de la comunicación y la cultura: en la búsqueda de algunas distinciones clasificatorias, en Luchessi, L. y Rodríguez M. G. (comps.) Fronteras Globales. Cultura, política y medios de comunicación.Buenos Aires: La Crujía,pp.275-301.

- Martini, S.(2000).Periodismo, noticia y noticiabilidad.Buenos Aires:Norma.

- Mastrini, G. y Becerra, M.(2006).Periodistas y Magnates. Estructura y concentración de las industrias culturales en América Latina. Buenos Aires: Prometeo.

- Palma, J.(2004).Escenas de la subalternidad politizada. Piquetes, saqueos y cacerolas: representaciones de lo popular politizado en la prensa gráfica. Tesina, Ciencias de la Comunicación,Facultad de Ciencias Sociales, Universidad de Buenos Aires.

- Palomino, H.; Bleynat, I.; Garro, S. y Giacomuzzi, C. (2011). "Cuestiones actuales sobre el universo de empresas recuperadas y las nuevas lógicas de agregación", OSERA,5, Buenos Aires: Observatorio Social sobre Empresas RecuperadasAutogestionadas.

- Perbellini, M.(2014).Empresas recuperadas: conflictividades, contradicciones inherentes y procesos de innovación e invención, ponencia presentada en Primer Encuentro Regional SudamericanoLa Economía de los trabajadores, Pigué, Provincia deBuenos Aires, 3 y 4 de octubre (paper).

- Ruggeri, A.(2014).Informe del IV relevamiento de Empresas Recuperadas en la Argentina, Programa Facultad Abierta, Facultad de Filosofía y Letras. Buenos Aires: Cooperativa Chilavert Artes gráficas. 
De Prácticas y discursos/ Universidad Nacional del Nordeste/ Centro de Estudios Sociales

- Settani, S.(2005).De la pueblada a losgrupos que violan permanentemente la ley: la representación de los piquetes construida por La Nación. Tesina, Ciencias de la Comunicación Social, Facultad de Ciencias Sociales, Universidad de Buenos Aires.

- Svampa, M. (2005).La Sociedad Excluyente. Buenos Aires: Taurus.

- Tuchman, G.(1986).La producción de la noticia. Barcelona: Gustavo Gili.

- Van Dijk, T.(1997).Análisis crítico de noticias,Racismo y análisis crítico de los medios. Buenos Aires: Paidós, pp.43-71.

- Verón, E.(1987).La semiosis social. Buenos Aires: Gedisa 